For the Journal of Colloid and Interface Science

\title{
Conductive-Probe Measurements with Nanodots of Free-base and Metallated Porphyrins
}

Xianglin Zhai ${ }^{\dagger}$ Neepa Malsi Kumari Kuruppu Arachchige, ${ }^{\dagger}$ Pedro Derosa,${ }^{\ddagger}$ and Jayne C. Garno ${ }^{* \dagger}$

${ }^{\dagger}$ Department of Chemistry, Louisiana State University, Baton Rouge, LA 70803, United States

${ }^{\ddagger}$ Physics Program, Louisiana Tech University, Ruston, LA 71272 and Department of Mathematics and Physics, Grambling State University, Grambling, LA 71245

*corresponding author: Jayne C. Garno

Phone: 225-578-8942

E-mail: jgarno@1su.edu

*address: $\quad$ Chemistry Department

Louisiana State University

232 Choppin Hall

Baton Rouge, LA 70803

FAX: 225-578-3458 


\begin{abstract}
The conductive properties of nanodots of model porphyrins were investigated using conductive-probe atomic force microscopy (CP-AFM). Porphyrins provide excellent models for preparing surface structures that can potentially be used as building blocks for devices. The conjugated, planar structure of porphyrins offers opportunities for tailoring the electronic properties. Two model porphyrins were selected for studies, 5,10,15,20tetraphenyl-21H,23H-porphine cobalt(II) (TPC) and its metal-free analog 5,10,15,20tetraphenyl-21H,23H-porphine (TPP). Nanodots of TPP and TPC were prepared within a dodecanethiol resist on gold using particle lithography. The nanopatterned surfaces exhibit millions of reproducible test structures of porphyrin nanodots. The porphyrin nanodots have slight differences in dimensions at the nanoscale, to enable size-dependent measurements of conductive properties. The size of the nanodots corresponds to $\sim 5-7$ layers of porphyrin. The conductivity along the vertical direction of the nanodots was measured by applying a bias voltage between the gold surface and a metal-coated AFM cantilever. The TPP nanodots exhibited semi-conductive profiles while the TPC nanodots exhibited profiles that are typical of a conductive film or molecular wire. The engineered nanostructures of porphyrins provide an effective platform for investigation and measurement of conductive properties.
\end{abstract}

KEYWORDS: conductive probe-atomic force microscopy, porphyrin, nanodots, conductivity, particle lithography 


\section{Introduction}

As components of miniaturized electronics, the conductive properties of porphyrin assemblies are relevant for potential applications. Well-developed synthetic routes can be employed to design the molecular structure of porphyrins, such as the placement and nature of the substituents and central metal ions [1-5]. Porphyrins have been investigated as possible building blocks in miniaturized electronics and photovoltaics [6-10].

Investigations of the conductivity of porphyrins have focused mainly on single molecules using scanning tunneling microscopy (STM) [11-16]. However, conductive probe-atomic force microcopy (CP-AFM) has become a practical tool for characterizing the conductive properties of organic films and assemblies in ambient conditions $[14,17-$ 23]. Early studies focused on applying CP-AFM to detect the conductivity of thiol selfassembled monolayers (SAMs) [21, 23-26]. An advantage of using CP-AFM to measure the conductive properties of organic films in comparison to STM studies is to avoid the effect of the gap between the tip and the sample [23, 27]. For AFM measurements, the tip is placed in direct contact with the sample, whereas STM measurements are made in noncontact mode, with the probe located a short distance from the sample. With AFM, a metal coated AFM probe is used as the top electrode which is placed at defined locations to record current-voltage (I-V) curves, while maintaining a controlled contact force and area between the tip and the sample.

Supramolecular assemblies of porphyrins such as nanorods, nanowires and nanotubes have been successfully synthesized and characterized [1, 28-32]. Nanorods of porphyrins can be prepared employing only the $\pi-\pi$ stacking interactions, or with 
additional intermolecular interactions such as van der Waals forces and metal-ligand coordination bonds [33-35]. Tubular nanostructures of porphyrin can be prepared using noncovalent interactions such as ionic bonds between two porphyrins with oppositely charged substituents [36,37] or a hybrid interaction including $\pi-\pi$ interactions and hydrogen bonding [38]. Wire-like nanostructures of porphyrins can be made by systematically adjusting the experimental conditions (immersion time and concentration; introducing surfactants; or using polar/nonpolar solvents $\left(\mathrm{H}_{2} \mathrm{O} / \mathrm{THF}\right)$ for controlling the aspect ratio of porphyrin nanorods $[28,39,40]$.

Studies of the photoconductivity of porphyrin nanorods of meso-tetrakis(4sulfonatophenyl)porphine (TPPS 4 ) were reported by Schmab, et al. that were characterized using an electrometer $[31,41]$. Nanorods of $\mathrm{TPPS}_{4}$ were investigated that had a well-defined height of $3.8 \pm 0.3 \mathrm{~nm}$ and lengths ranging from 0.2 to $2 \mu \mathrm{m}$. The conductivity of nanorods of $\mathrm{TPPS}_{4}$ was also studied using scanning tunneling microscopy (STM) in a UHV environment by Friesen, et al. [32]. The charge-carrier mobility of multiple wire-like porphyrin nanostructures formed with octaethylporphyrin containing selected metals was investigated by So, et al. using an electrometer [40]. The conductivities of zinc(II) metalloporphyrins were evaluated using single-wall carbon nanotubes for CP-AFM measurements [9]. Isolated nanoclusters of zinc porphyrin were prepared in a thiol matrix and the electronic properties of the nanoclusters were characterized with UHV-STM $[42,43]$.

A limitation for improving the sensitivity of scanning-probe based conductive measurements is the availability of well-defined test platforms of sample materials. Porphyrins are difficult to pattern, because when assembled on surfaces the molecules do 
not form chemisorbed or covalent bonds with metal substrates. Rather, porphyrins tend to self-associate and bind together in a stacked arrangement to form overlapping layers of molecules to adopt a coplanar, physisorbed configuration on surfaces [44]. Emerging practical approaches for preparing regularly shaped nanostructures with particle lithography have been demonstrated with organothiols and organosilanes [45-47].

For approaches based on particle lithography, samples can be prepared with a surface mask of monodisperse latex or silica spheres. After the particles are removed, exposed sites of the substrate are available for further steps of chemical reactions [46, 48, 49]. Combining solution immersion with particle lithography provides a practical strategy to prepare porphyrin nanostructures. Millions of nanopores can be generated within organothiol or organosilane SAMs [50-53]. The nanopores provide a spatially confined nano-sized container for depositing porphyrins. The array of porphyrin nanodots enable multiple test structures for reproducible CP-AFM measurements. Methyl-terminated dodecanethiol was used as a resistive matrix surrounding the nanodots, minimizing nonspecific adsorption over areas between nanopores.

We have developed a strategy to use alkanethiol nanopatterns prepared by particle lithography to spatially confine and define the locations for porphyrin deposition. In our study, the conductivity of porphyrin nanodots prepared within a resistive matrix of dodecanethiol on $\mathrm{Au}(111)$ was measured using CP-AFM. The molecules selected were 5,10,15,20-tetraphenyl-21H,23H-porphyrin cobalt(II) (TPC) and 5,10,15,20-tetraphenyl$21 \mathrm{H}, 23 \mathrm{H}-$ porphyrin (TPP), as symmetric model structures of free-base and metallated porphyrins. The structures of TPP and TPC are shown in Scheme S1. Properties of materials at the nanoscale do not necessarily scale to the established trends of bulk 
samples. At the molecular level, one would predict that addition of a metal atom to the core of a porphyrin macrocycle would increase the conductivity of the molecule. Our experiments enable comparisons of the conductive properties of the two molecular systems, as well as provide size dependent measurements of conductance.

\section{Experimental Section}

\subsection{Materials}

Reagents such as 5,10,15,20-tetraphenyl-21H,23H-porphine cobalt(II) (TPC), 5,10,15,20-tetraphenyl-21H,23H-porphine (TPP) ,1-dodecanethiol and dichloromethane were acquired from Sigma Aldrich (St. Louis, MO) and used as received. Ethanol (200 proof) was obtained from Pharmco-AAPER (Shelbyville, KY) and used as the diluent for preparing solutions of alkanethiols. Dichloromethane was obtained from Sigma Aldrich (St. Louis, MO) for dissolving porphyrins. Gold pellets (99.99\% purity) were purchased from Ted Pella (Redding, CA). Ruby muscovite mica was obtained from S\&J Trading Inc., (Glen Oaks, NY).

\subsection{Preparation of porphyrin solutions}

To prepare porphyrin solutions for making patterns of nanostructures on substrates, TPC and TPP were dissolved in dichloromethane $\left(10^{-3} \mathrm{M}\right)$ and then diluted with ethanol to a concentration of $10^{-7} \mathrm{M}$. Samples of each porphyrin were prepared by immersing a nanopatterned substrate in dilute solutions for $30 \mathrm{~h}$.

\subsection{Preparation of template-stripped gold}

The process for preparing template-stripped gold (TSG) is described in a previous publication [53]. A thin layer $(150 \mathrm{~nm})$ of gold was thermally deposited onto mica at $10^{-7}$ Torr using a high-vacuum thermal evaporator (Angstrom Engineering Inc., Kitchener, 
OR). Freshly cleaved Ruby muscovite mica was preheated to $350^{\circ} \mathrm{C}$ prior to gold deposition using quartz lamps at the back of the sample holder. The gold pellets were thermally evaporated and deposited onto mica at $10^{-7}$ Torr with a deposition rate of $3 \AA$ s. After deposition, the gold films were annealed at $365^{\circ} \mathrm{C}$ in vacuum for $30 \mathrm{~min}$ and then cooled to room temperature before removal from the deposition chamber. The method for mechanical stripping was previously described by Hegner et al. [54]. First, the gold substrates and glass slides were cleaned with deionized water and placed into a UVozone generator for $30 \mathrm{~min}$. Epoxy (EPO-TEK, Billerica, MA) was applied onto the clean glass slides and then attached onto the gold to make a glass-gold-mica "sandwich." The sample was then heated at $150^{\circ} \mathrm{C}$ for $2 \mathrm{~h}$ to anneal the epoxy. After annealing, the glass pieces were carefully peeled from the mica to produce TSG with atomically flat surfaces. 2.4 Preparation of porphyrin nanostructures by immersion particle lithography

The preparation of nanopores within alkanethiol SAMs has been described previously [53]. A graphical description of the method for preparing patterns of nanodots is outlined in Supporting Information, Scheme S2. Surface masks for immersion particle lithography were made by depositing monodisperse silica spheres $(500 \mathrm{~nm}$ diameter, Thermo-Fisher Scientific, Waltman, MA) onto freshly stripped TSG. For cleaning the spheres, $300 \mu \mathrm{L}$ of a suspension of silica spheres was transferred into a plastic microcentrifuge tube and centrifuged for $20 \mathrm{~min}$ at $20,000 \mathrm{rpm}$. A solid pellet of the spheres formed at the bottom of the centrifuge tube. The supernatant was decanted and then deionized water was added to the centrifuge tube for rinsing. The spheres were resuspended into deionized water by vortex mixing. The rinsing step was repeated four times. A volume of $15 \mu \mathrm{L}$ of the mesosphere suspension was deposited onto TSG and 
dried in ambient conditions to form surface masks. Before immersing into thiol solutions, the surface masks were placed into an oven at $150^{\circ} \mathrm{C}$ for $12 \mathrm{~h}$. This step is important for preventing the silica spheres from being displaced from the substrate when immersed in solution. The samples were cooled to room temperature $\left(25^{\circ} \mathrm{C}\right)$, then immersed into a $10^{-3}$ M ethanolic dodecanethiol solution for $24 \mathrm{~h}$. Dodecanethiol SAMs formed on the exposed areas of the TSG surfaces around the areas protected by the spheres. Next, the spheres were removed in a further step by sonication in ethanol. Nanopores were fabricated within a matrix film of dodecanethiol. The center-to-center spacing between the nanopores matches the diameter of the mesosphere masks (i.e. $500 \mathrm{~nm}$ ). In the final step, the nanopores were submerged into a $10^{-7} \mathrm{M}$ solution of TPC or TPP in dichloromethane/ethanol (1:10000 vol) for $30 \mathrm{~h}$ to produce porphyrin nanodots. Samples were rinsed with ethanol and dried under argon. The uncovered areas of gold substrate that had been masked by spheres provided well-defined surface sites for directing the subsequent attachment and growth of porphyrin nanodots.

\subsection{Atomic Force Microscopy}

A model 5420 scanning probe microscope (Keysight Technologies, Santa Rosa, CA) installed with PicoView v1.12 software was used for scanning probe studies. Images were acquired using tapping mode in ambient conditions. Imaging was accomplished with rectangular shaped, ultrasharp silicon tips that have an aluminum reflex coating, with an average spring constant of $48 \mathrm{~N} / \mathrm{m}$ (Nanoscience Instruments, Phoenix, AZ). The scan rate was 1 line/s. Electrical measurements on porphyrin nanodots were performed with the same instrument equipped with conductive nosecone assembly $(0.1 \mathrm{nA} / \mathrm{V}$ sensitivity). Conductive tips with a platinum coating (PPP-CONTPt, Nanosensors, 
Neuchatel, Switzerland) were used to acquire I-V curves. Gwyddion v. 2.32 was used to process and analyze the digital images [55].

\section{Results and discussion}

Porphyrins with and without a central metal ion were patterned, 5,10,15,20tetraphenyl-21H,23H-porphyrin (TPP) and 5,10,15,20-tetraphenyl-21H,23H-porphyrin cobalt(II) (TPC) using steps of solution immersion of a gold substrate masked with silicon spheres. Protocols for patterning porphyrin nanodots and nanorods on gold substrates were based on immersion particle lithography. With particle lithography, individual nanodots of porphyrins were spatially isolated into well-defined arrangements within a matrix film of a nanopatterned thiol monolayer with particle lithography. The nanodots of TPP and TPC attached to areas of the gold substrate, surrounded by an insulating film of dodecanethiol. Nanopores within a methyl-terminated alkanethiol matrix were filled with porphyrin nanodots by immersing the substrates with dodecanethiol nanopatterns into solutions of TPP or TPC. The porphyrin nanodots were not completely monodisperse, and exhibited sufficient differences in dimensions at the nanoscale to enable size-dependent I-V measurements.

\subsection{Substrates with dodecanethiol nanopatterns}

Example images of the arrangement of nanopores within a matrix of dodecanethiol are presented in Fig. 1. Broad regions of the sample exhibit areas of uncovered circles of substrate. A methyl-terminated self-assembled monolayer (SAM) formed surrounding the $500 \mathrm{~nm}$ spheres on gold substrates after $20 \mathrm{~h}$ immersion into a 
solution of dodecanethiol. When the surface mask of $500 \mathrm{~nm}$ spheres was rinsed away, nanopores within dodecanethiol were generated as shown in Fig. 1.
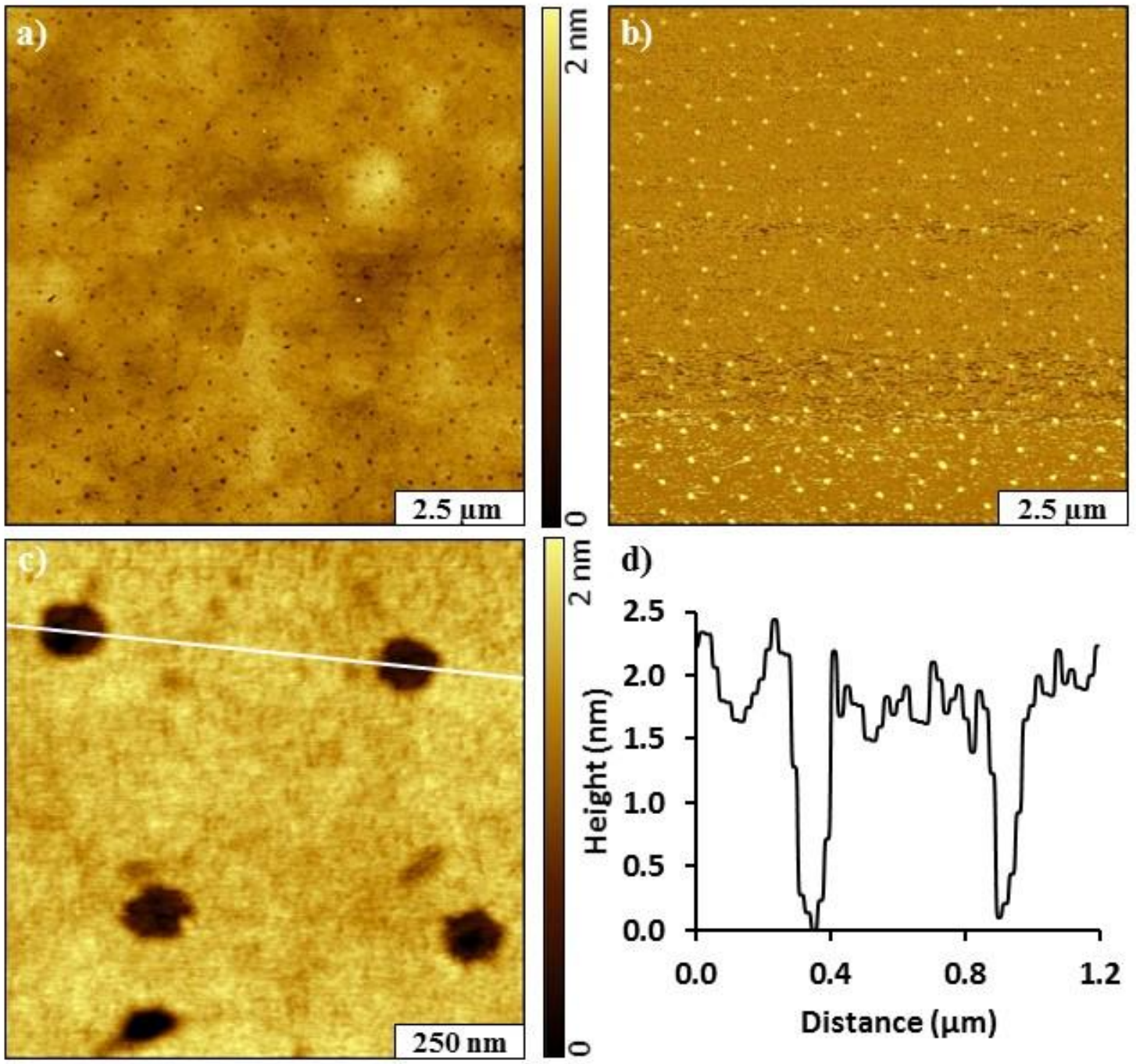

Fig. 1. Nanopores within dodecanethiol formed on gold. (a) Topography and (b) corresponding phase image; (c) zoom-in view; (d) cursor profile for the line in $c$.

The arrangement of nanopores is shown throughout areas of the sample in Fig. 1a. The nanopores arrange in a pattern reflecting the way the spheres assembled on the surface. The distance between the nanopores measures $500 \mathrm{~nm}$, which corresponds to the size of the silica spheres used as surface mask. Within a $10 \times 10 \mu \mathrm{m}^{2}$ frame, there are 223 
nanopores scaling to a surface density of $10^{8}$ nanopores $/ \mathrm{cm}^{2}$. The overall area of the uncovered nanopores measured $\sim 1 \%$ of the entire frame. The differences in surface chemistry between the nanopores and surrounding matrix are evidenced with the simultaneously acquired phase image (Fig. 1b). Brighter contrast indicates the region of uncovered gold substrate within the nanopores. These areas were protected from deposition of thiols by the spheres of the particle mask and provide sites for depositing porphyrins. The actual shapes of the nanopores is revealed in a zoom-in view (Fig. 1c). Circular shaped nanopores provide a template area for further steps of patterning TPP and TPC. The depth of the holes measured $1.7 \pm 0.2 \mathrm{~nm}$, shown with a representative cursor profile in Fig. 1d.

\subsection{Nanopatterned dots of porphyrins}

The areas of uncovered gold substrate within the methyl-terminated thiol SAM were used as nano-sized containers to deposit TPP and TPC. Millions of nanostructures on the surface test platforms enable the evaluation of the reproducibility of multiple measurements, as well as the visualization of whether the sample was damaged by electrical pulses during I-V measurements. The morphologies of the nanostructures formed either by TPP or TPC were characterized by tapping mode AFM in ambient conditions. Dot-like surface structures of porphyrins formed primarily within the nanopores, indicating that the methyl-terminated SAM of dodecanethiol was an effective resist. 

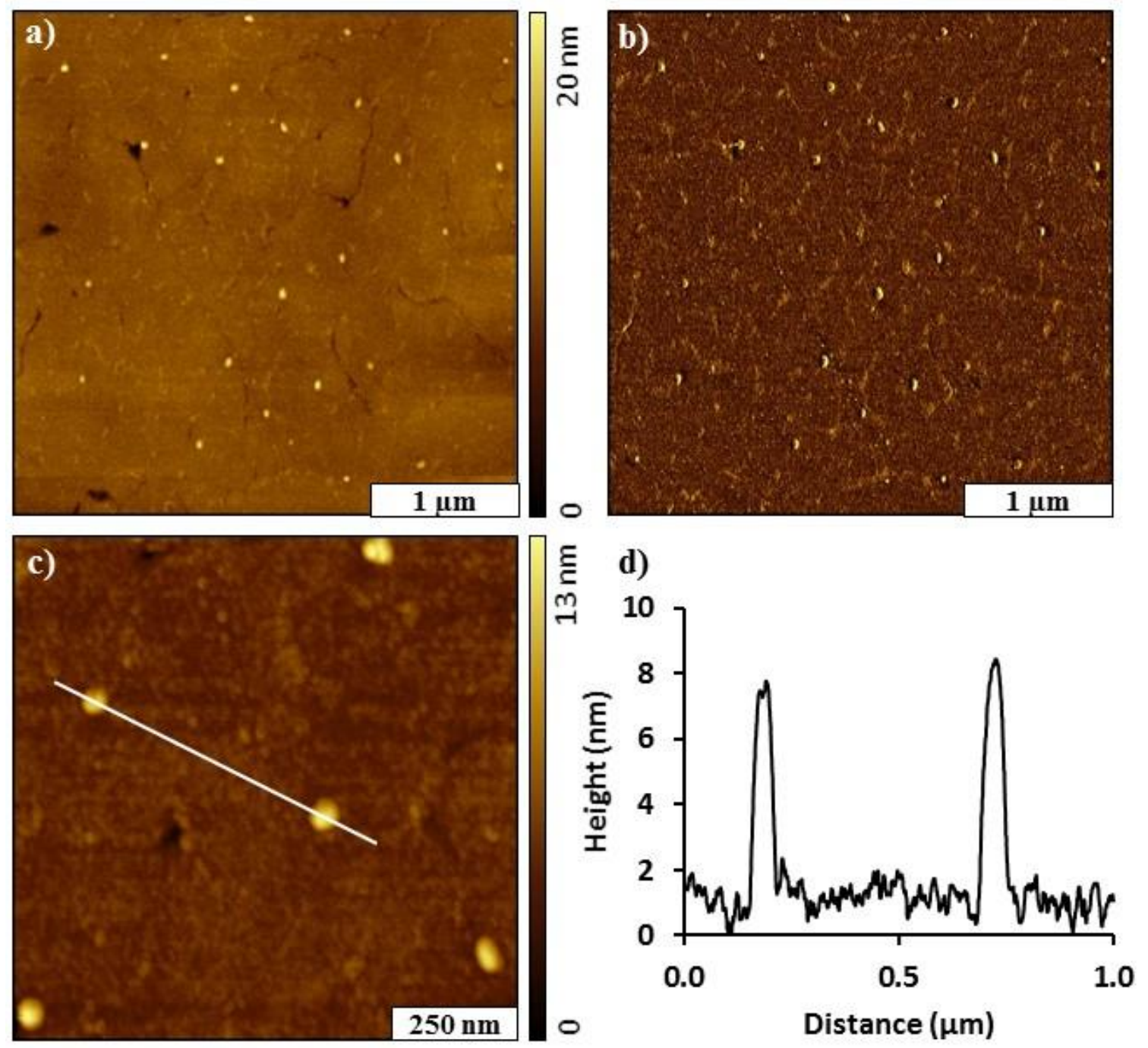

Fig. 2. Nanodots of TPP within dodecanethiol (representative views). (a) Topography and (b) corresponding phase image; (c) zoom-in view; (d) height profile across two nanodots in $c$.

An array of nanodots of TPP (Fig. 2) was formed by immersion of a nanopore template in $10^{-7} \mathrm{M}$ solution for $30 \mathrm{~h}$. The TPP nanodots are regularly arranged in a periodic pattern with a few areas of missed nanopores. A few defects of gold substrate under the thiol SAM are resolved in the broader areas of the topography image of Fig. $2 \mathrm{a}$. At the nanoscale, characteristics of the $\mathrm{Au}(111)$ substrate such as roughness, scars and gold terraces affect the packing of the spheres used for particle lithography to create a 
few defects. The distance between neighboring nanodots can be controlled by selecting spheres with different diameters as the surface mask. Further details of the size, placement and shapes of the TPP nanodots are presented in the corresponding phase image (Fig. 2b). A close-up of the nanodots is viewed in the topography image of Fig. 2c. The nanodots measure $8 \mathrm{~nm}$ in height and are spaced $500 \mathrm{~nm}$ apart (Fig. 2d). The nanostructures provide an excellent test platform for further studies with conductive probe AFM, because there are multiple, isolated nanocrystals for replicate measurements.
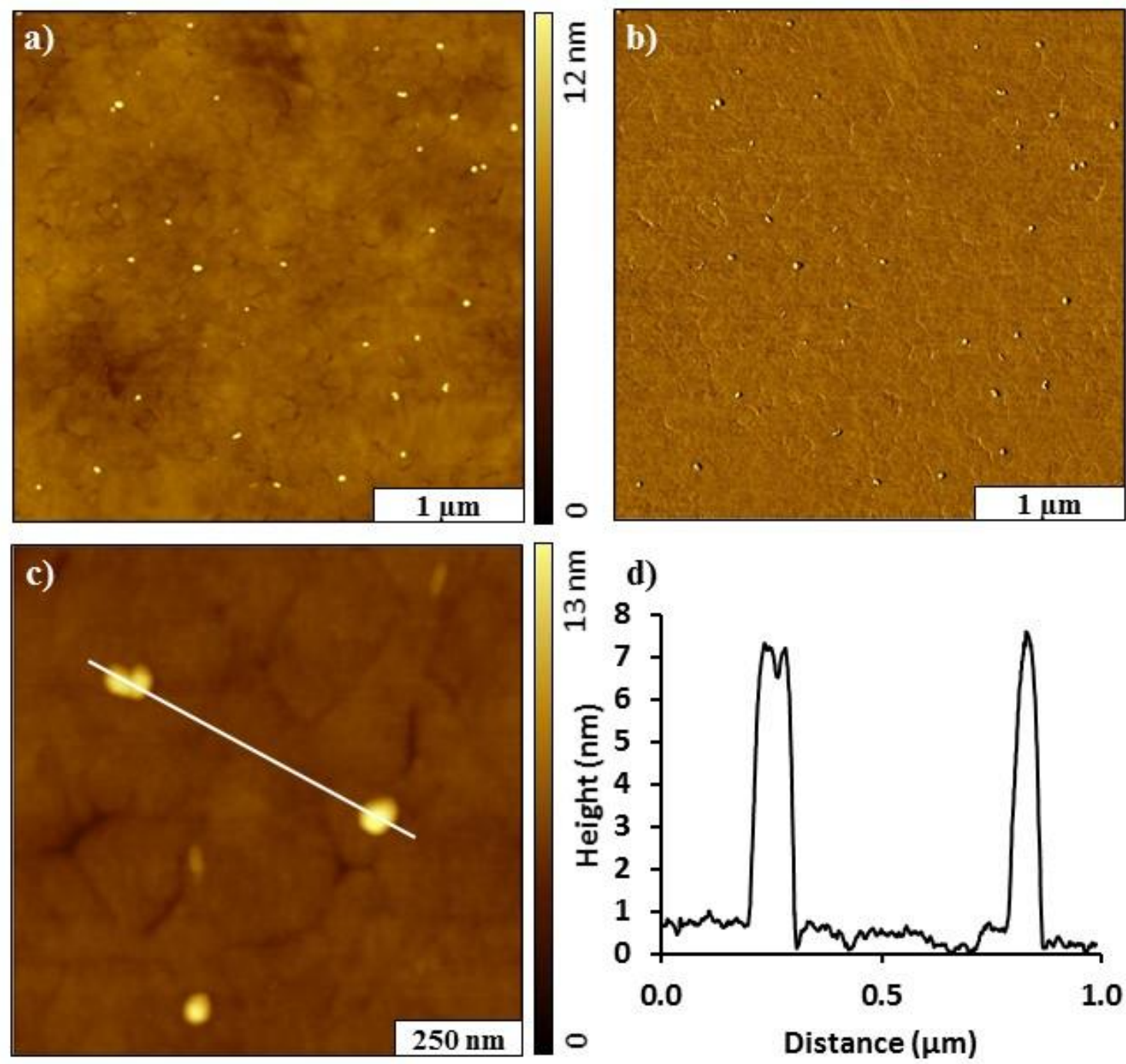

Fig. 3. Nanodots of TPC within dodecanethiol. (a) Topography and (b) corresponding phase image; (c) zoom-in view; (d) height profile across two nanodots in $\boldsymbol{c}$. 
An array of TPC nanodots also was formed by immersion of a nanopatterned dodecanethiol/Au template in a $10^{-7} \mathrm{M}$ solution of TPC for $30 \mathrm{~h}$. The overall shapes and surface arrangement of TPC nanodots is indistinguishable from that of the TPP sample. Nanodots of TPC formed inside the nanopores (Fig. 3), and mostly did not assemble on the areas of dodecanethiol. The selectivity of the TPC binding to areas of the nanopores is evident in the simultaneously acquired phase image (Fig. 3b). The areas of dodecanethiol matrix SAM appear to be free of adsorbates or nanoparticles. The detailed morphology of a few TPC nanodots is shown in Fig. 3c. The example nanodots measure $7 \pm 0.5 \mathrm{~nm}$ in height (Fig. 3d).
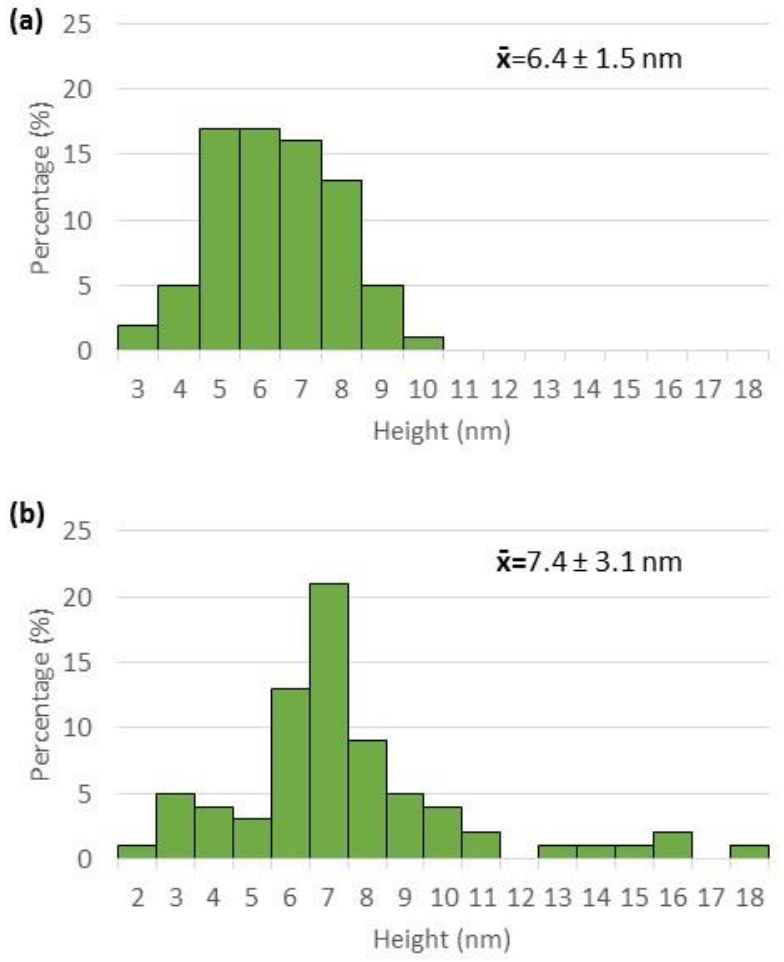

Fig. 4. Heights of the nanodots of TPP and TPC. (a) Height distribution of TPP nanodots $(n=76)$. (b) Height of TPC nanodots $(n=73)$. 
Within the same sample, the sizes of the TPP and TPC nanodots are slightly polydisperse, at the nanoscale. This is because the surface template of nanopores within dodecanethiol was not perfect at such small dimension. Slight imperfections in the sizes of the spheres or substrate defects cannot be perfectly controlled at the scale of less than $10 \mathrm{~nm}$. The sizes of nanodots formed by TPP or TPC are compared in Fig. 4. The average height of the TPP nanodots measured $6.4 \pm 1.5 \mathrm{~nm}$ while the TPC nanodots measured 7.4 $\pm 3.1 \mathrm{~nm}$. With a two sample t-test, the heights of the TPP and TPC nanodots are not significantly different with equal viabilities. With the consideration of the depth of nanopores, the thickness of the nanodots measured $\sim 7 \mathrm{~nm}$, indicating multiple layers of porphyrin were deposited within the nanopores. The dimensions correspond approximately to 5-7 layers or stacks of porphyrins in a coplanar arrangement. The estimate is based on the height of a coplanar orientation of a planar TPP to be $\sim 1.1 \mathrm{~nm}$. The methyl-terminated areas of the surrounding matrix of dodecanethiol provide an effective resist layer, minimizing the non-specific adsorption of porphyrins in areas surrounding the nanopores. Porphyrins have a planar structure that tends to bind to flat surfaces in a stacked arrangement, with the macrocycle of the molecules oriented parallel to the substrate. The nature of surface attachment is physisorption, which is a relatively weak attachment for nanopatterning protocols. There are strong $\pi-\pi$ interactions that drive macrocycles to bind together in a stacked arrangement, like a stack of coins.

\subsection{Conductive measurements with porphyrin nanostructures}

The conductivity of the nanodots was measured in the vertical direction through the layers of porphyrin using conductive probe AFM. A platinum coated AFM tip was used as an electrode and was placed on the nanodots to establish a closed circuit loop 
with the gold substrate for the current flowing through the nanodot. A single measurement was made for each nanodot, because the surface contact became oxidized after applying voltage. As a test, we evaluated the changes in measurements with multiple successive measurements, and have included additional examples of I-V spectra in Supporting Information, Figs. S1 and S2.
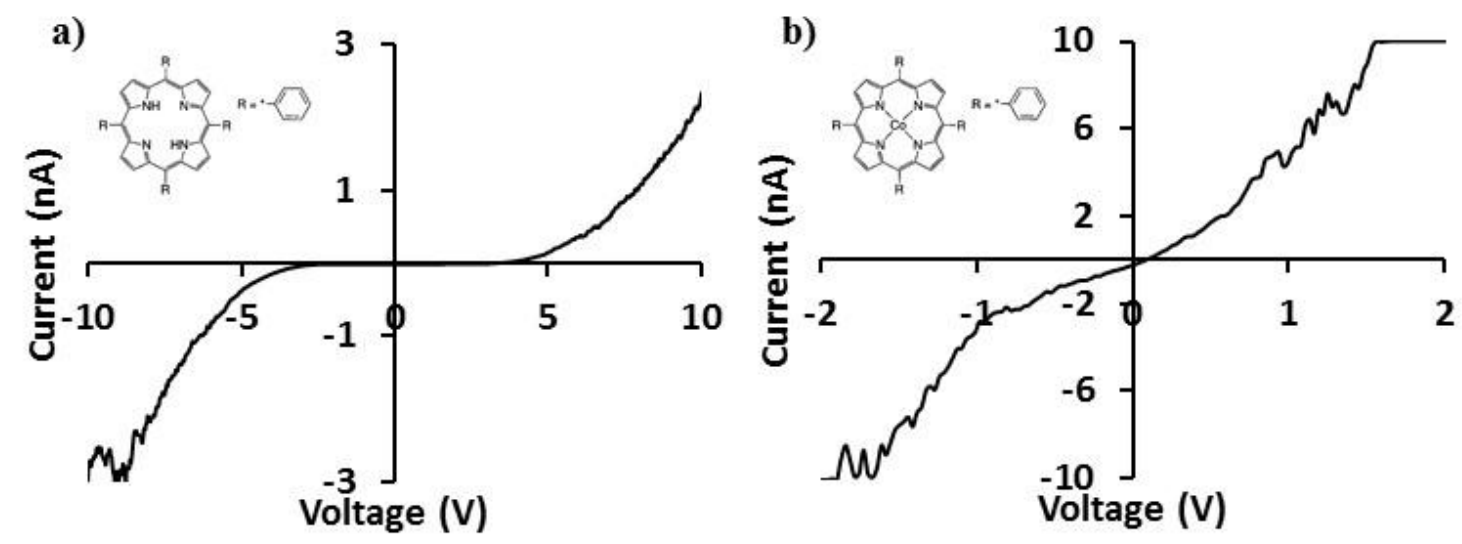

Fig. 5. Example I-V curves for individual nanodots of (a) TPP and (b) TPC.

Representative I-V curves of TPP nanodots and TPC nanodots are shown in Fig. 5. To minimize the impact of the loading force and geometry of the tip on the current through the nanostructures, $[25,56]$ the same tip was used to collect all the I-V curves with controlled load less than $1 \mathrm{nN}$. The TPP nanodots exhibit a typical I-V profile of a semi-conductive material (Fig. 5a). The maximum current measured through the TPP nanodot is $3 \mathrm{nA}$ at $10 \mathrm{~V}$. The current gradually decreases to zero when the voltage decreases from $10 \mathrm{~V}$ to $4 \mathrm{~V}$ in both negative and positive ranges. In between $-4 \mathrm{~V}$ to $+4 \mathrm{~V}$, the TPP nanodots are relatively nonconductive. In contrast, the TPC nanodots show better conductivity in a relatively small voltage range, which is $-2 \mathrm{~V}$ to $+2 \mathrm{~V}$. In this range, the maximum current through a TPC nanodot measured $10 \mathrm{nA}$ at $2 \mathrm{~V}$. The cobalt core in the 
molecular structure has a significant role in the conductive properties. The current gradually decreases to zero when the voltage was decreased from $2 \mathrm{~V}$ to $0 \mathrm{~V}$ in both the negative and positive ranges. The TPC nanodots with a cobalt ion coordinated to the macrocycle (Fig. 5b) has a more conductive, wire-like profile than the semiconductive free-base porphyrin, TPP (Fig. 5a). The magnitude of the measured currents and voltages are quite distinct depending on whether the nanodots contain a metal ion.

The porphyrin nanodots are an excellent model surface structure for conductive probe measurements. The sizes of the nanodots have slight variability at the nanoscale, which enables size-dependent analysis. Further example I-V curves collected from the TPP and TPC nanodots are presented in Supporting Information, Fig. S3. Representative I-V curves from nanodots of TPC with different height across the range $(4 \mathrm{~nm}, 10 \mathrm{~nm}, 18)$ is added as Fig. S4.

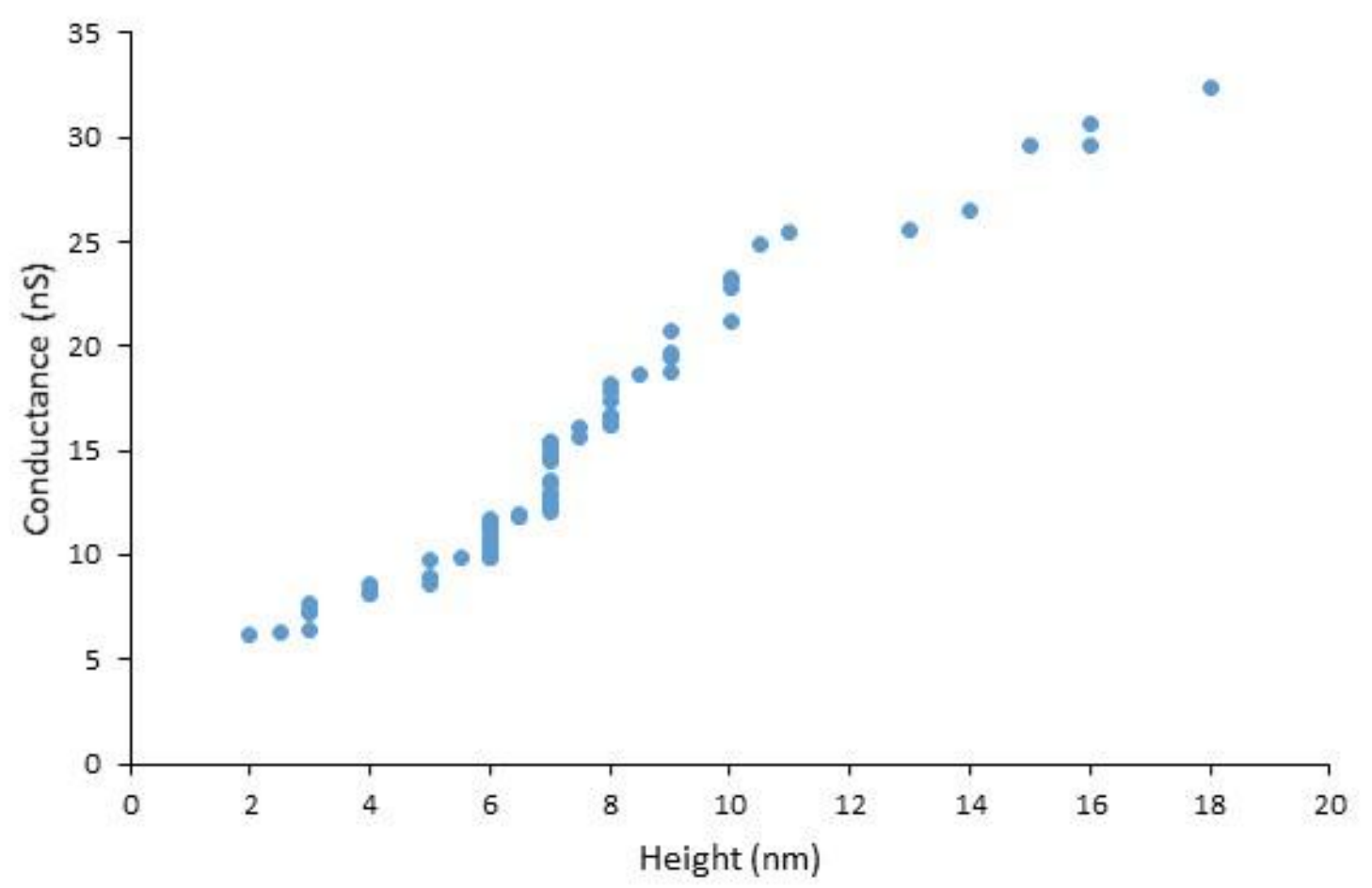

Fig. 6. Heights versus the measured conductance of TPC nanodots. $(n=78)$ 
Further analysis of the effects of the size of nanodots can be evaluated, as shown in Fig. 6. The heights of the nanodots were plotted versus conductance measurements from 78 TPC nanodots. The heights of the nanodots ranged from 2 to $20 \mathrm{~nm}$. A clear trend is apparent that shows greater conductance for taller nanostructures of TPC. The conductance of the TPC nanodots was measured from the slopes of I-V curves. The conductivity of the nanodot formed by TPC molecules can be expressed as the equation $(1)$,

$$
\delta=\mathrm{G} \times \mathrm{h} / \mathrm{A}
$$

where, $\delta$ is conductivity $(\mathrm{S} / \mathrm{m}), \mathrm{h}$ is the height of the nanodots and A is the transverse area of the nanodots $\left(\mathrm{nm}^{2}\right)$. The average conductivity of the TPP nanodots is $0.046 \mathrm{~S} / \mathrm{m}$.

The trend for conductance increasing with the size of the nanodots relates directly to the stacking of additional molecules into a compact assembly. With fewer molecules, the smaller nanodots have a lower conductance, whereas the larger nanodots include additional cobalt ions. With the addition of more cobalt-coordinated molecules, the nanodots exhibit increasing conductance.

\section{Conclusions}

A new test platform is introduced for making conductive probe measurements of regularly-shaped, small clusters of porphyrins. Surface structures of nanodots of porphyrins were prepared in nanopores formed within dodecanethiol/Au(111). Dot-like nanostructures of porphyrins with and without a metal center were self-assembled within nanopores prepared by immersion particle lithography. The conductivity profiles were measured and compared using CP-AFM for 3-10 nm nanodots of cobalt-coordinated and free-base porphyrins. Distinct I-V profiles were exhibited with TPC and TPP nanodots 
that were chosen as model systems. The key difference between the structures of the two selected porphyrins is the cobalt core. For TPP nanodots, profiles that are typical of semiconductive films were observed. With cobalt-coordinated TPC nanodots, wire-like conductive profiles were exhibited. As might be predicted, the conductivity of TPC nanodots is greater than that of TPP nanodots. The approach described demonstrates the feasibility for sensitive measurements of the conductivity of multiple, replicate nanodots with CP-AFM. The discussion and data herein focus on two particular porphyrins, however the measuring technique can be applied to a wide range of thin film materials. As further molecular systems are studied in the future, a better understanding of the mechanisms of organic conduction can be anticipated at the nanoscale with this technique.

\section{Acknowledgments}

The authors gratefully acknowledge financial support from the National Science Foundation Louisiana Alliance for Simulation-Guided Materials Applications (LASiGMA) (EPS-1003897). We thank Zheng Xue for helpful discussions of statistical methods. 
Graphical abstract

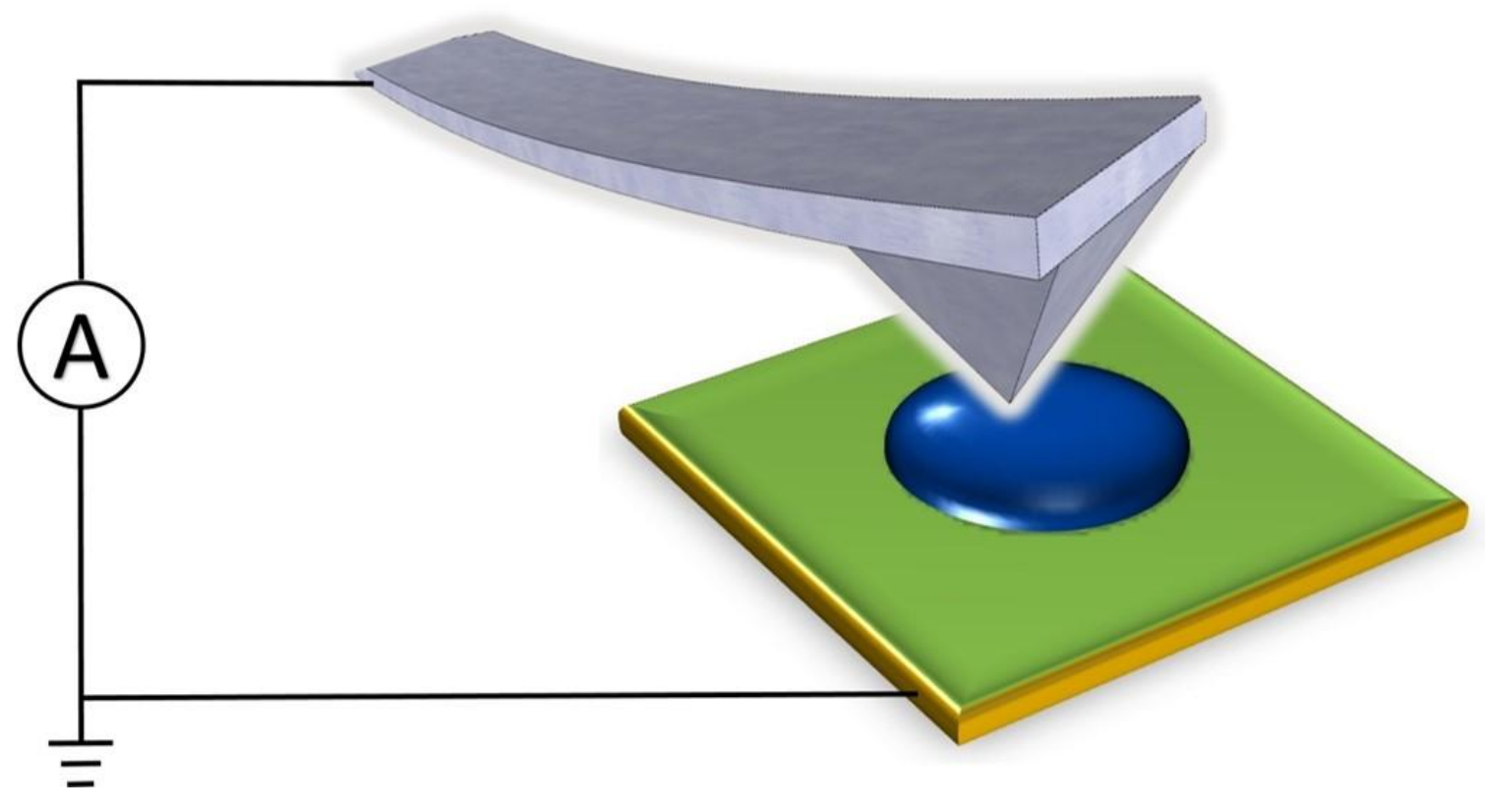




\section{References}

[1] A.C. Aragones, N. Darwish, W.J. Saletra, L. Perez-Garcia, F. Sanz, J. Puigmarti-Luis, D.B. Amabilino, I. Diez-Perez, Highly conductive single-molecule wires with controlled orientation by coordination of metalloporphyrins, Nano Lett. 14 (2014) 4751-6.

[2] N.V. Bhupathiraju, W. Rizvi, J.D. Batteas, C.M. Drain, Fluorinated porphyrinoids as efficient platforms for new photonic materials, sensors, and therapeutics, Organic \& biomolecular chemistry 14 (2015) 389-408.

[3] T. Hasobe, Photo- and electro-functional self-assembled architectures of porphyrins, Physical chemistry chemical physics : PCCP 14 (2012) 15975-87.

[4] K. Ladomenou, V. Nikolaou, G. Charalambidis, A.G. Coutsolelos, "Click"-reaction: An alternative tool for new architectures of porphyrin based derivatives, Coord Chem Rev 306 (2016) 1-42.

[5] C.J. Medforth, Z. Wang, K.E. Martin, Y. Song, J.L. Jacobsen, J.A. Shelnutt, Self-assembled porphyrin nanostructures, Chem. Commun. (2009) 7261-7277.

[6] M.S. Dyer, A. Robin, S. Haq, R. Raval, M. Persson, J. Klimeš, Understanding the Interaction of the Porphyrin Macrocycle to Reactive Metal Substrates: Structure, Bonding, and Adatom Capture, ACS Nano 5 (2011) 1831-1838.

[7] Y. Wan, A. Stradomska, S. Fong, Z. Guo, R.D. Schaller, G.P. Wiederrecht, J. Knoester, L. Huang, Exciton Level Structure and Dynamics in Tubular Porphyrin Aggregates, J Phys Chem C 118 (2014) 24854-24865.

[8] J. Cai, H. Chen, J. Huang, J. Wang, D. Tian, H. Dong, L. Jiang, Controlled self-assembly and photovoltaic characteristics of porphyrin derivatives on a silicon surface at solid-liquid interfaces, Soft matter 10 (2014) 2612-8.

[9] H. Tanaka, T. Yajima, T. Matsumoto, Y. Otsuka, T. Ogawa, Porphyrin molecular nanodevices wired using single-walled carbon nanotubes, Adv. Mater. 18 (2006) 1411-+.

[10] M. Jurow, A.E. Schuckman, J.D. Batteas, C.M. Drain, Porphyrins as Molecular Electronic Components of Functional Devices, Coord. Chem. Rev. 254 (2010) 2297-2310.

[11] S. Vijayaraghavan, D. Ecija, W. Auwarter, S. Joshi, K. Seufert, A.P. Seitsonen, K. Tashiro, J.V. Barth, Selective Supramolecular Fullerene-Porphyrin Interactions and Switching in SurfaceConfined C-60-Ce(TPP)(2) Dyads, Nano Lett. 12 (2012) 4077-4083.

[12] L. Grill, K.H. Rieder, F. Moresco, S. Stojkovic, A. Gourdon, C. Joachim, Exploring the interatomic forces between tip and single molecules during STM manipulation, Nano Lett. 6 (2006) 2685-2689.

[13] S. Battacharyya, A. Kibel, G. Kodis, P.A. Liddell, M. Gervaldo, D. Gust, S. Lindsay, Optical Modulation of Molecular Conductance, Nano Lett. 11 (2011) 2709-2714.

[14] W. Xi, W. Zhang, B.K. An, P.L. Burn, J.J. Davis, Tunnelling conductance of vectorial porphyrin monolayers, J. Mater. Chem. 18 (2008) 3109-3120.

[15] S.C. Yan, Z.J. Ding, N. Xie, H.Q. Gong, Q. Sun, Y. Guo, X.Y. Shan, S. Meng, X.H. Lu, Turning on and off the Rotational Oscillation of a Single Porphine Molecule by Molecular Charge State, ACS Nano 6 (2012) 4132-4136.

[16] S. Joshi, F. Bischoff, R. Koitz, D. Ecija, K. Seufert, A.P. Seitsonen, J. Hutter, K. Diller, J.I. Urgel, H. Sachdev, J.V. Barth, W. Auwarter, Control of Molecular Organization and Energy Level Alignment by an Electronically Nanopatterned Boron Nitride Template, ACS Nano 8 (2014) 430442.

[17] P.F. Siles, C.C.B. Bufon, D. Grimm, A.R. Jalil, C. Mende, F. Lungwitz, G. Salvan, D.R.T. Zahn, H. Lang, O.G. Schmidt, Morphology and local transport characteristics of metalloporphyrin thin films, Org Electron 15 (2014) 1432-1439. 
[18] X.Y. Wang, G.C. Wang, K.M. Lewis, High rectification ratios of Fe-porphyrin molecules on Au facets, Mater. Chem. Phys. 136 (2012) 190-195.

[19] C.Y. Mou, W.L. Yuan, I.S. Tsai, E.A. O'Rear, H. Barraza, Conductance measurement by twoline probe method of polypyrrole nano-films formed on mica by admicellar polymerization, Thin Solid Films 516 (2008) 8752-8756.

[20] L.A. Bumm, J.J. Arnold, T.D. Dunbar, D.L. Allara, P.S. Weiss, Electron transfer through organic molecules, J. Phys. Chem. B 103 (1999) 8122-8127.

[21] D.J. Wold, C.D. Frisbie, Fabrication and characterization of metal-molecule-metal junctions by conducting probe atomic force microscopy, J. Am. Chem. Soc. 123 (2001) 5549-5556.

[22] M. Salmeron, G. Neubauer, A. Folch, M. Tomitori, D.F. Ogletree, P. Sautet, Viscoelastic and Electrical-Properties of Self-Assembled Monolayers on Au(111) Films, Langmuir 9 (1993) 36003611.

[23] D.J. Wold, C.D. Frisbie, Formation of metal-molecule-metal tunnel junctions: Microcontacts to alkanethiol monolayers with a conducting AFM tip, J. Am. Chem. Soc. 122 (2000) 2970-2971.

[24] X.D. Cui, X. Zarate, J. Tomfohr, O.F. Sankey, A. Primak, A.L. Moore, T.A. Moore, D. Gust, G. Harris, S.M. Lindsay, Making electrical contacts to molecular monolayers, Nanotechnology 13 (2002) 5-14.

[25] G. Leatherman, E.N. Durantini, D. Gust, T.A. Moore, A.L. Moore, S. Stone, Z. Zhou, P. Rez, Y.Z. Liu, S.M. Lindsay, Carotene as a molecular wire: Conducting atomic force microscopy, J. Phys. Chem. B 103 (1999) 4006-4010.

[26] D.J. Wold, R. Haag, M.A. Rampi, C.D. Frisbie, Distance Dependence of Electron Tunneling through Self-Assembled Monolayers Measured by Conducting Probe Atomic Force Microscopy: Unsaturated versus Saturated Molecular Junctions, J Phys Chem B 106 (2002) 2813-2816.

[27] Q. Ferreira, L. Alcacer, J. Morgado, Stepwise preparation and characterization of molecular wires made of zinc octaethylporphyrin complexes bridged by 4,4'-bipyridine on HOPG, Nanotechnology 22 (2011) 435604.

[28] M.H. Hoang, Y. Kim, S.J. Kim, D.H. Choi, S.J. Lee, High-Performance Single-Crystal-Based Organic Field-Effect Transistors from pi-Extended Porphyrin Derivatives, Chem.-Eur. J. 17 (2011) 7772-7776.

[29] B.K. Kang, N. Aratani, J.K. Lim, D. Kim, A. Osuka, K.H. Yoo, Electrical transport properties and their reproducibility for linear porphyrin arrays, Mater. Sci. Eng. C 26 (2006) 1023-1027.

[30] D.H. Yoon, S.B. Lee, K.H. Yoo, J. Kim, J.K. Lim, N. Aratani, A. Tsuda, A. Osuka, D. Kim, Electrical conduction through linear porphyrin arrays, J. Am. Chem. Soc. 125 (2003) 1106211064.

[31] A.D. Schwab, D.E. Smith, B. Bond-Watts, D.E. Johnston, J. Hone, A.T. Johnson, J.C. de Paula, W.F. Smith, Photoconductivity of Self-Assembled Porphyrin Nanorods, Nano Lett. 4 (2004) 12611265.

[32] B.A. Friesen, B. Wiggins, J.L. McHale, U. Mazur, K.W. Hipps, Differing HOMO and LUMO Mediated Conduction in a Porphyrin Nanorod, J. Am. Chem. Soc. 132 (2010) 8554-8556.

[33] T. Hasobe, H. Oki, A.S.D. Sandanayaka, H. Murata, Sonication-assisted supramolecular nanorods of meso-diaryl-substituted porphyrins, Chem. Commun. (2008) 724.

[34] A.S.D. Sandanayaka, Y. Araki, T. Wada, T. Hasobe, Structural and Photophysical Properties of Self-Assembled Porphyrin Nanoassemblies Organized by Ethylene Glycol Derivatives, J Phys Chem C 112 (2008) 19209-19216.

[35] Y.N. Gao, X.M. Zhang, C.Q. Ma, X.Y. Li, J.Z. Jiang, Morphology-Controlled Self-Assembled Nanostructures of 5,15-Di[4-(5-acetylsulfanylpentyloxy)phenyl]porphyrin Derivatives. Effect of Metal-Ligand Coordination Bonding on Tuning the Intermolecular Interaction, J. Am. Chem. Soc. 130 (2008) 17044-17052. 
[36] Z.C. Wang, C.J. Medforth, J.A. Shelnutt, Porphyrin nanotubes by ionic self-assembly, J. Am. Chem. Soc. 126 (2004) 15954-15955.

[37] Z.C. Wang, C.J. Medforth, J.A. Shelnutt, Self-metallization of photocatalytic porphyrin nanotubes, J. Am. Chem. Soc. 126 (2004) 16720-16721.

[38] T. Kojima, R. Harada, T. Nakanishi, K. Kaneko, S. Fukuzumi, Porphyrin nanotubes based on self-assembly of $\mathrm{Mo}(\mathrm{V})$-dodecaphenylporphyrin complexes and inclusion of Mo-oxo clusters: Synthesis and characterization by X-ray crystallography and transmission electron microscopy, Chem. Mater. 19 (2007) 51-58.

[39] S.J. Lee, J.T. Hupp, S.T. Nguyen, Growth of narrowly dispersed porphyrin nanowires and their hierarchical assembly into macroscopic columns, J. Am. Chem. Soc. 130 (2008) 9632-+.

[40] M.-H. So, V.A.L. Roy, Z.-X. Xu, S.S.-Y. Chui, M.-Y. Yuen, C.-M. Ho, C.-M. Che, Controlled SelfAssembly of Functional Metal Octaethylporphyrin $1 \mathrm{D}$ Nanowires by Solution-Phase Precipitative Method, Chemistry - An Asian Journal 3 (2008) 1968-1978.

[41] A.D. Schwab, D.E. Smith, C.S. Rich, E.R. Young, W.F. Smith, J.C. de Paula, Porphyrin Nanorods, The Journal of Physical Chemistry B 107 (2003) 11339-11345.

[42] Y.H. Chan, A.E. Schuckman, L.M. Perez, M. Vinodu, C.M. Drain, J.D. Batteas, Synthesis and characterization of a thiol-tethered tripyridyl porphyrin on Au(111), J Phys Chem C 112 (2008) 6110-6118.

[43] A.E. Schuckman, B.W. Ewers, L.H. Yu, J.P.C. Tome, L.M. Perez, C.M. Drain, J.G. Kushmerick, J.D. Batteas, Utilizing Nearest-Neighbor Interactions To Alter Charge Transport Mechanisms in Molecular Assemblies of Porphyrins on Surfaces, J Phys Chem C 119 (2015) 13569-13579.

[44] G. Lu, Y. Chen, Y. Zhang, M. Bao, Y. Bian, X. Li, J. Jiang, Morphology Controlled SelfAssembled Nanostructures of Sandwich Mixed (Phthalocyaninato)(Porphyrinato) Europium Triple-Deckers. Effect of Hydrogen Bonding on Tuning the Intermolecular Interaction, J Am Chem Soc 130 (2008) 11623-11630.

[45] J.-R. Li, J.C. Garno, Elucidating the Role of Surface Hydrolysis in Preparing Organosilane Nanostructures via Particle Lithography, Nano Lett. 8 (2008) 1916-1922.

[46] J.-R. Li, B.R. Lewandowski, S. Xu, J.C. Garno, Detecting the Magnetic Response of Iron Oxide Capped Organosilane Nanostructures Using Magnetic Sample Modulation and Atomic Force Microscopy, Anal. Chem. 81 (2009) 4792-4802.

[47] C.K. Saner, K.L. Lusker, Z.M. LeJeune, W.K. Serem, J.C. Garno, Self-assembly of octadecyltrichlorosilane: Surface structures formed using different protocols of particle lithography. (Invited for theme issue: "Self-assembly at solid surfaces"), Beilstein J. Nanotechnol. 3 (2012) 114-122.

[48] K.L. Lusker, J.-J. Yu, J.C. Garno, Particle Lithography with Vapor Deposition of Organosilanes: A Molecular Toolkit for Studying Confined Surface Reactions in Nanoscale Liquid Volumes, Thin Solid Films 7 (2011) 5223-5229.

[49] T. Tian, Z.M. LeJeune, J.C. Garno, Directed surface assembly of 4-(chloromethyl)phenyltrichlorosilane: Self-polymerization within spatially-confined sites of Si(111) viewed by atomic force microscopy, Langmuir 29 (2013) 6529-6536.

[50] L. Isa, K. Kumar, M. Müller, J. Grolig, M. Textor, E. Reimhult, Particle Lithography from Colloidal Self-Assembly at Liquid-Liquid Interfaces, ACS Nano 4 (2010) 5665-5670.

[51] L. Lu, S.H. Lahasky, D. Zhang, J.C. Garno, Directed Growth of Polymer Nanorods Using Surface-Initiated Ring-Opening Polymerization of N-Allyl N-Carboxyanhydride, ACS applied materials \& interfaces 8 (2016) 4014-22.

[52] L.E. Englade-Franklin, C.K. Saner, J.C. Garno, Spatially selective surface platforms for binding fibrinogen prepared by particle lithography with organosilanes, Interface focus 3 (2013) 20120102. 
[53] X. Zhai, H.J. Lee, T. Tian, T.R. Lee, J.C. Garno, Nanoscale lithography mediated by surface self-assembly of 16-[3,5-bis(mercaptomethyl)phenoxy]hexadecanoic acid on $\mathrm{Au}(111)$ investigated by scanning probe microscopy, Molecules 19 (2014) 13010-26.

[54] M. Hegner, P. Wagner, G. Semenza, Ultralarge atomically flat template-stripped Au surfaces for scanning probe microscopy, Surf. Sci. 291 (1993) 39-46.

[55] D. Necas, P. Klapetek, Gwyddion: An open-source software for SPM data analysis, Cent. Eur. J. Phys. 10 (2012) 181-188.

[56] M.J. Loiacono, E.L. Granstrom, C.D. Frisbie, Investigation of charge transport in thin, doped sexithiophene crystals by conducting probe atomic force microscopy, J. Phys. Chem. B 102 (1998) 1679-1688. 